\title{
In vitro and in silico studies of MDM2/MDMX isoforms predict Nutlin-3A sensitivity in well/de-differentiated liposarcomas
}

\author{
Fabio Bozzi ${ }^{1}$, Elena Conca ${ }^{1}$, Erik Laurini², Paola Posocco², Alessandra Lo Sardo ${ }^{3}$, Genny Jocollè \\ Roberta Sanfilippo ${ }^{4}$, Alessandro Gronchi ${ }^{5}$, Federica Perrone ${ }^{1}$, Elena Tamborini ${ }^{1}$, Giuseppe Pelosi ${ }^{1}$, \\ Marco A Pierotti ${ }^{6}$, Roberta Maestro ${ }^{3,7}$, Sabrina Pricl ${ }^{2,7}$ and Silvana Pilotti ${ }^{1,7, *}$
}

The molecular marker of well-differentiated/de-differentiated liposarcomas is MDM2 gene amplification coupled with protein overexpression and wild-type TP53. MDMX is a recently identified MDM2 homolog and its presence in this tumor is unexplored. Our aim was to investigate the role of full-length MDM2 and MDMX proteins and their isoforms in surgical specimens of well-differentiated/de-differentiated liposarcomas in view of Nutlin-3A (a MDM2 inhibitor) treatment. Frozen and matched formalin-fixed, paraffin-embedded material from surgical specimens was examined by means of:

(1) fluorescence in situ hybridization to determine MDM2 and MDMX gene copy numbers; (2) RT-PCR and densitometry to analyze alternative splicing forms of $m d m 2$ and $m d m x$; (3) immunoblotting and immunohistochemistry to assess the corresponding translated proteins; and (4) in vitro and in silico assays to determine their affinity for Nutlin-3A. All these cases showed MDM2 gene amplification with an MDMX disomic pattern. In all cases, the full-length $m d m 2$ transcript was associated with the $m d m 2-b$ transcript, with ratios ranging from 0.07 to 5.6 , and both were translated into protein; $m d m x$ and $m d m x$-s were co-transcripted, with ratios ranging from 0.1 to 5.6. MDMX-S was frequently more upregulated than MDMX at both transcriptional and protein level. Each case showed different amounts of $m d m 2, m d m 2-b, m d m x$, and $m d m x-s$ transcripts and the corresponding proteins. In vitro assays showed that Nutlin-3A was ineffective against MDM2-B and was unable to disrupt the MDMX/TP53 and MSMX-S/TP53 complexes. Molecular simulations confirmed these in vitro findings by showing that MDM2 has high Nutlin-3A affinity, followed by MDMX-S, MDMX, and MDM2-B. Nutlin-3A is predicted to be a good therapeutic option for well-differentiated/de-differentiated liposarcomas. However, our findings predict heterogeneous responses depending on the relative expression of $m d m 2, m d m 2-b, m d m x$, and $m d m x-s$ transcripts and proteins.

Laboratory Investigation (2013) 93, 1232-1240; doi:10.1038/labinvest.2013.107; published online 9 September 2013

KEYWORDS: MDM2; MDMX; molecular modeling; Nutlin-3A; TP53

Well-differentiated/de-differentiated (WD/DD) liposarcomas (LPS) account for $40 \%$ of all liposarcomas. With a few exceptions, ${ }^{1,2}$ the DD component is defined as a usually abrupt and generally a phenotypic time-dependent transition to a non-lipogenic sarcoma.

WD/DD LPS characteristically involve the retroperitoneum and are characterized by a high rate of local recurrences. The inability of even aggressive surgery to obtain negative margins leads to high local failure rates, worsening the long-term prognosis of WD/DD patients and favors the use of multiple treatment modalities albeit with limited benefit. ${ }^{3,4}$

The molecular hallmark of WD/DD LPS is MDM2 gene amplification coupled with protein overexpression and wild-type TP53, ${ }^{5-7}$ which provides the rationale supporting the therapeutic potential of the MDM2 antagonist Nutlin-3A. MDM2 is an E3 ubiquitin ligase that, in addition to targeting TP53 for proteasomal degradation, blocks TP53

\footnotetext{
${ }^{1}$ Laboratory of Experimental Molecular Pathology, Department of Pathology, Fondazione IRCCS, Istituto Nazionale dei Tumori, Milan, Italy; ${ }^{2}$ Molecular Simulation Engineering Laboratory, DICAMP, University of Trieste, Trieste, Italy; ${ }^{3}$ Experimental Oncology 1, CRO National Cancer Institute, Aviano. Pordenone, Italy; ${ }^{4}$ Adult Sarcoma Unit, Department of Cancer Medicine, Fondazione IRCCS, Istituto Nazionale dei Tumori, Milan, Italy; ${ }^{5}$ Department of Surgical Pathology, Fondazione IRCCS, Istituto Nazionale dei Tumori, Milan, Italy and ${ }^{6}$ Scientific Directorate, Fondazione IRCCS, Istituto Nazionale dei Tumori, Milan, Italy

*Correspondence: Dr S Pilotti, Department of Pathology, Fondazione IRCCS, Istituto Nazionale per lo Studio e la Cura dei Tumori, Via G. Venezian 1, 20133 Milano, Italy. E-mail: silvana.pilotti@istitutotumori.mi.it
}

${ }^{7}$ Senior co-authors.

Received 29 July 2013; accepted 4 August 2013 
transcriptional activity. Nutlin-3A binds MDM2 in the TP53 binding pocket and interferes with MDM2-directed TP53 degradation; this induces TP53 stabilization and activation, and leads to cell cycle arrest, the inhibition of cell growth, and apoptosis, which may also be mediated by upregulation of the CDKN1A (p21) transcript. ${ }^{8}$ Interestingly, it has been reported that tumors overexpressing gene amplification-mediated MDM2 are the most sensitive to MDM2 inhibitors, at least at preclinical level. ${ }^{9}$ However, the therapeutic potential of Nutlin-3A may be limited because it is known that full-length MDM2 may be accompanied by a number of splice variant transcripts (arising from alternative exon splicing) in different tumor histotypes and, as these variants may not retain either wholly or partially the full TP53 binding site required for Nutlin-3A binding, they may not be targeted. A number of splicing forms lacking the TP53/MDM2 binding site have been found in WD/DD LPS. ${ }^{10,11}$

In addition to gene amplification, MDM2 overexpression may also be mediated by the SNP309 ( $\mathrm{T}$ to $\mathrm{G}$ transition) polymorphism in the promoter region of MDM2 that has recently been associated with WD/DD LPS. ${ }^{12}$ According to Seyfried et $a l,^{13}$ the presence SNP309 predicts a high degree of sensitivity to Nutlin-3A treatment in chronic lymphocytic leukemia cells, although this was not subsequently confirmed. ${ }^{14}$

A further factor that may affect drug sensitivity is MDMX, a negative regulator of TP53 that forms heterocomplexes with MDM2 and is essential for the MDM2-mediated polyubiquitinilation of TP53. ${ }^{15}$ Despite the close similarity between the TP53 binding domains of MDM2 and MDMX, Nutlin-3A fails to inhibit the TP53/MDMX complex in vivo and in vitro, ${ }^{16,17}$ possibly because its binding affinity for MDMX is less than that for MDM2. ${ }^{17}$

Like MDM2, MDMX also has splice variants, the most widely investigated of which is MDMX-S, a truncated form of MDMX that retains the TP53 binding domain responsible for TP53 inhibition. ${ }^{18}$ On the basis of recent findings, the MDMX protein levels may be closely regulated by posttranslational modifications using the alternative splicing of $m d m x$ mRNA. In this way, the ratio between the $m d m x$-s splice variant and the full-length $m d m x$ becomes the means by which $m d m x$-s mRNA expression reduces that of the full-length MDMX protein, ${ }^{19}$ and it has recently been reported that the reduced MDMX protein expression mediated by $m d m x / m d m x-s$ is associated with a worse prognosis in patients with osteosarcoma. ${ }^{20}$

The role of MDMX and MDMX-S in WD/DD LPS has not yet been defined. It has been reported that MDMX protein expression is very low in WD/DD LPS cell lines, ${ }^{21}$ and $M D M X$ gene amplification has been described in association with MDM2 co-amplification in one WD/DD LPS surgical specimen. ${ }^{22}$ However, in a recently published series of 28 $\mathrm{WD} / \mathrm{DD}$ cases, only one carried a low level of MDMX gene amplification (between three- and six-fold). ${ }^{12}$ Finally, there are no published data regarding the presence of MDMX-S in
WD/DD LPS, although it has been identified in 70\% of soft tissue sarcomas. ${ }^{22}$

We have now found that MDM2 and full-length MDMX, together with their MDM2-B and MDMX-S isoforms, are expressed and translated in WD/DD LPS surgical specimens. Furthermore, the findings of our in vitro and in silico experiments show that they contribute differently to Nutlin-3A sensitivity and resistance.

\section{MATERIALS AND METHODS Patient Samples}

We studied both frozen and formalin-fixed, paraffin-embedded (FFPE) samples of 8 DD and 5 WD LPS obtained from 12 patients ( 6 with retroperitoneal and 6 with nonretroperitoneal lesions, Supplementary Table 1) who underwent surgical resection in different locations (Supplementary Table 1). All of the FFPE samples included both WD and DD components, but the only frozen sample to contain both came from patient 8 (see Supplementary Table 1, samples 8a and $8 \mathrm{~b}$ ). As expected, the results of all of the analyses of this matched pair of samples were similar.All of the patients gave their written informed consent.

All of the samples underwent fluorescence in situ hybridization (FISH), DNA extraction and SNP309 sequencing, RNA extraction and reverse transcription, full-length $m d m 2, m d m 2-a, m d m 2-b$, and $m d m 2-c$ transcript amplification, and PCR product sub-cloning and sequencing as described in Supplementary Materials and Methods.

\section{Densitometry}

The PCR products were run on a $1.5 \%$ agarose gel, and the images acquired through the Gel-Doc apparatus (Bio-Rad, Segrate, Italy) were analyzed using Quantity One software (Bio-Rad) in accordance with the manufacturer's instructions. Further details are given in the legend to Figure 1.

\section{MDM2 and MDMX Immunohistochemistry (IHC)}

Representative sections of the WD and DD components were selected for each case and immunophenotyped for MDM2 and MDMX. The anti-MDM2 (ab-1, cat. \#OP46, Oncogene Research Products, San Diego, CA, USA) and anti-HdmX/ MDM4 antibodies (cat. \#A300.287A, Bethyl Laboratories, Montgomery, TX, USA) were respectively diluted 1:20 and $1: 800$, and the antigen was retrieved using $1 \mathrm{mmol} / \mathrm{l}$ EDTA, $\mathrm{pH} 8$, in an autoclave at $95^{\circ} \mathrm{C}$ for $30 \mathrm{~min}(\mathrm{MDM} 2)$ or $6 \mathrm{~min}$ (MDMX). All of the antibodies were developed as previously described. $^{23}$

\section{Ectopic Expression of MDMX-S}

The transfections into HEK293T cells and analyses of ectopic MDMX-S expression were performed as previously described $^{24}$ using a mouse monoclonal anti-MDMX antibody (cat. \#M0445, MX-82, Sigma, St Louis, MO, USA). Anti- $\beta$-tubulin antibody (cat. \#sc-1904, H235, 


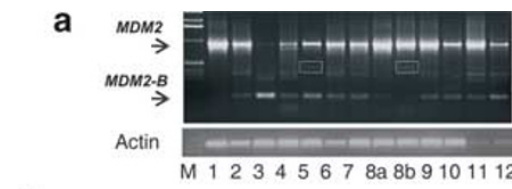

b

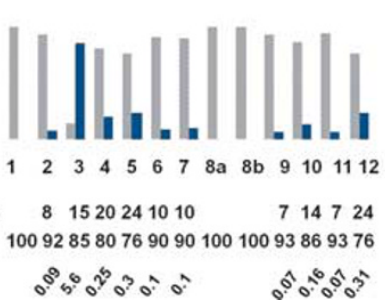

C

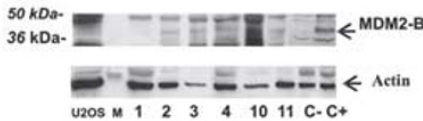

d

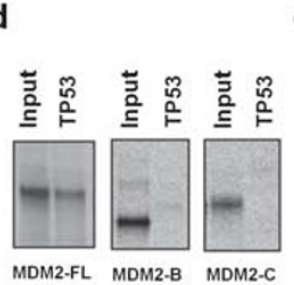

e

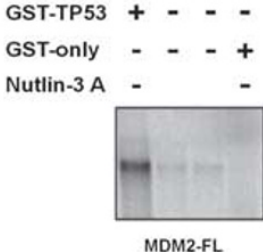

Figure 1 (a) Representative $m d m 2$ WD/DD LPS RT-PCR results. The bands corresponding to full-length $m d m 2$, $m d m 2-b$, and $m d m 2-c$ are indicated by the arrows and boxes. Given the complex pattern of the $m d m 2$ transcripts, all of the PCR products were cloned and sequenced. With the exception of full-length $m d m 2, m d m 2-b$, and $m d m 2-c$, all of the amplified bands were artifactual reverse transcription products that were not considered in the subsequent analyses. (b) Densitometry of the $m d m 2$ (gray columns) and $m d m 2-b$ (blue columns) transcripts, expressed as the rate of full-length and alternative transcripts with respect to the total (full-length plus splicing forms $=100 \%$ ). The ratios were obtained by dividing the $m d m 2-b$ rate by the corresponding full-length $m d m 2$ rate. (c) WB analysis of six representative WD/DD samples. The arrow indicates the band corresponding to MDM2-B $(40 \mathrm{kDa})$. The MDM2-B used as positive $(\mathrm{C}+)$ and negative controls $(\mathrm{C}-)$, were obtained by means of an in vitro translation reaction as described in Supplementary Materials and Methods and Supplementary Figure 2. The MDM2-disomic osteosarcoma cell line U2OS was used as a further MDM2-Bnegative control. (d) Full-length (FL) MDM2 (but not MDM2-B and MDM2-C) interacts with TP53 in vitro. GST pull-down assay of GST-TP53 with IVTMDM2-FL, MDM2-B, and MDM2-C. The input represents 10\% of the reaction before the binding assay. (e) Nutlin-3A displaces the TP53/MDM2-FL interaction. GST pull-down assays of GST-TP53 and IVT MDM2-FL in the absence or presence of increasing amounts of Nutlin-3A. The last lane represents the control reaction of MDM2-FL against GST alone.

sc-9104, Santa Cruz Biotechnology, Santa Cruz, CA, USA) was used for normalization.

\section{Western Blotting}

The frozen material was analyzed by means of western blotting (WB) as previously described. ${ }^{23}$ To detect MDM2, MDM2-B, and MDM2-C, we used a rabbit polyclonal antiMDM2 antibody raised against the MDM2 N-terminal domain and diluted 1:200 (N-20, cat. \#sc-813, Santa Cruz Biotechnology); MDMX-S was detected using a mouse monoclonal anti-MDMX antibody raised against the MDMX N-terminal domain and diluted 1:1000 (MX-82, Sigma).

\section{In Vitro GST Pull-Down Assay}

The GST pull-down experiments were performed as previously described. ${ }^{24}$ Briefly, full-length MDM2 and MDM2 B, MDM2 C, MDMX, and MDMX-S proteins were in vitro translated (IVT) with $\left[{ }^{35} \mathrm{~S}\right]$-methionine (Perkin Elmer, Monza, Italy) using TNT System (Promega, Milan, Italy). The IVT proteins ( $5 \mu \mathrm{l}$ of the IVT reaction) were preincubated with the indicated amounts of Nutlin-3A in $500 \mu \mathrm{l}$ PLB (20 mM Tris-Cl, pH 8.0, $150 \mathrm{mM} \mathrm{NaCl}, 1 \mathrm{mM}$ EDTA, $0.1 \%$ Nonidet-P40, $1 \mathrm{mM}$ PMSF plus complete protease inhibitors (Roche, Milan, Italy) for $2 \mathrm{~h}$ at $4{ }^{\circ} \mathrm{C}$, and then allowed to react with $4 \mu \mathrm{g}$ of either GST/hTP53 or GST recombinant proteins in the presence of $40 \mu \mathrm{l}$ of $50 \%$ slurry glutathione sepharose $4 \mathrm{~B}$ resin (GE Healthcare, Milan, Italy) for $2 \mathrm{~h}$ at $4{ }^{\circ} \mathrm{C}$. The amount of Nutlin-3A used in these experiments $(0,80$, and $400 \mu \mathrm{M})$ was established experimentally according to $\mathrm{Hu}$ et $a l^{17}$ and Poyurovsky et al. ${ }^{25}$
Efficient inhibition of the interaction between full-length TP53 and full-length MDM2 was used as a positive control. After extensive washings, the bound proteins were separated by means of SDS-PAGE, after which the gels were stained with Coomassie Brilliant Blue, dried and exposed to X-ray film (Kodak, Cinisello Balsamo, Italy).

\section{Molecular Modeling}

The three-dimensional models of MDM2, MDMX, and all of their splicing variants were constructed using validated homology techniques. ${ }^{26}$ Binding affinity between all of the proteins and Nutlin-3A was estimated using wellestablished molecular dynamics (MD) experiments ${ }^{27}$ based on the molecular mechanics/Poisson-Boltzmann surface area (MM/PBSA) methodology. ${ }^{28}$ Advanced steered molecular dynamics (SMD) experiments ${ }^{29}$ were also performed to confirm the MM/PBSA-derived affinity values by studying the unbinding process of Nutlin-3A from MDM2, MDMX, and all of their spicing variants (see Supplementary Materials and Methods for full details).

\section{RESULTS \\ MDM2}

FISH

FISH analysis revealed that all of the examined specimens showed MDM2 gene amplification in both the WD and DD components (not shown).

\section{SNP309}

In our case material (Supplementary Table 1), the frequency of $\mathrm{G} / \mathrm{G}, \mathrm{G} / \mathrm{T}$, and $\mathrm{T} / \mathrm{T}$ alleles was respectively $53 \%, 8 \%$ and 
$39 \%$, which is in line with the values recently found in another series of WD/DD samples. ${ }^{12}$

\section{MDM2 Alternative Transcript Detection and Quantification}

Specific primers were designed for the co-amplification of fulllength $m d m 2 \mathrm{cDNA}$ and its transcript variants, and the PCR products were cloned and sequenced (Supplementary Figure 1). We concentrated on $m d m 2-a$, $m d m 2-b$, and $m d m 2-c$ because, in addition to being translated into the corresponding proteins, ${ }^{11}$ they are also characterized by the loss of the $\mathrm{N}$-terminal TP53 binding site and are therefore not expected to be targeted by Nutlin-3A.

Full-length $m d m 2$ was present in all of the samples; $m d m 2-b$ was identified in six of the eight DD and four of the five WD samples, and $m d m 2-c$ in one of the eight $\mathrm{DD}$ and one of the five WD samples (Figure 1a). No transcripts corresponding to $m d m 2-a$ were found in any of the WD or DD samples.

The relative amounts of $m d m 2, m d m 2-b$, and $m d m 2-c$ transcripts were evaluated by means of semiquantitative PCR and densitometry on $1.5 \%$ agarose gels. In DD sample 3 (Figure $1 \mathrm{~b}$ ), the $m d m 2-b / m d m 2$ ratio was 5.6 (corresponding to $85 \%$ of $m d m 2-b$ and $15 \%$ of $m d m 2$ transcripts); in the remaining cases, the ratio ranged from 0.07 to 0.3 (corresponding to 7 and $24 \%$ of $m d m 2-b$ transcripts); $m d m 2-c$ accounted for only $1 \%$ of the total $m d m 2$ transcripts ( $m d m 2-c$ $/ m d m 2$ ratio $=0.01)$. The high $m d m 2-b / m d m 2$ ratio observed in DD sample 3 was not accompanied by any morphological changes in comparison with the other samples evaluated and currently, the patient is alive and with no evident disease after six years from surgery.

These findings, which confirm previously published data, ${ }^{10,11}$ showed that $m d m 2-b$ is the most commonly expressed $m d m 2$ alternative transcript in WD/DD LPS, and that its amount varies widely from case to case.

\section{MDM2 and MDM2-B IHC and WB Analyses}

IHC and WB experiments were used to confirm the presence of the corresponding proteins. Using an antibody against fulllength MDM2, its nuclear localization was demonstrated by means of IHC in matched FFPE samples of both the WD and DD components (the same as those used for the FISH analysis) (data not shown). WB revealed a protein of $40 \mathrm{kDa}$, corresponding to the molecular weight of MDM2-B $\mathrm{B}^{11}$ (Figure 1c; also see Supplementary Materials and Methods and Supplementary Figure 2) but, in line with the small amount of transcript, no band corresponding to the expected weight of MDM2-C protein was observed. Taken together, these results show that both full-length $m d m 2$ and the $m d m 2-b$ splicing variant are translated into proteins in WD/DD LPS samples.

\section{Sensitivity of TP53/MDM2 Complexes to Nutlin-3A}

The ability of Nutlin-3A to inhibit the interaction of TP53 with MDM2 was evaluated using in vitro GST pull-down assays. Briefly, the different splicing forms of MDM2 (MDM2 full length, MDM2-B, and MDM2-C) were in vitro translated and incubated with GST-TP53 in the absence or presence of Nutlin-3A, and the purified GST-TP53/MDM2 complexes were resolved by SDS-PAGE and quantified. As expected, Nutlin-3A displaced the binding of TP53 to MDM2 in a dosedependent manner. In keeping with the absence of the canonical TP53 binding domain, the ability of MDM2-B and MDM2-C to bind GST-TP53 was impaired (Figures 1d and e) and, consequently, Nutlin-3A had no effect on these variants.

\section{MDM2, MDM2-B, and MDM2-C Molecular Modeling}

MD simulations carried out using the entire MDM2 protein in complex with Nutlin-3A showed that the affinity $\left(\Delta \mathrm{G}_{\text {bind }}\right)$ of the oncogene for the inhibitor was $-9.11 \mathrm{kcal} / \mathrm{mol}$ (Table 1). The corresponding $\mathrm{IC}_{50}$ value of $210 \mathrm{nM}$ is in line with the available range of experimental data (100$300 \mathrm{nM}),{ }^{8,17,30,31}$ and confirms that Nutlin-3A rather nicely fits the full-length MDM2 binding pocket (Figures $2 \mathrm{a}$ and $\mathrm{b}$ ). The use of MM/PBSA to investigate Nutlin-3A in complex with MDM2-B (Figure 2c) and MDM2-C (Figure 2d) showed that MDM2-C is capable of binding Nutlin-3A to a very low extent $\left(\Delta \mathrm{G}_{\text {bind }}=-7.03 \mathrm{kcal} / \mathrm{mol}, \quad \mathrm{IC}_{50}=7.1 \mu \mathrm{M}, \Delta \Delta \mathrm{G}_{\text {bind }}\right.$ $=-2.08 \mathrm{kcal} / \mathrm{mol}$, Table 1$)$, and the affinity of MDM2-B was as much as two orders of magnitude less: $\Delta \mathrm{G}_{\text {bind }}=-6.01$ $1 \mathrm{kcal} / \mathrm{mol}, \quad \mathrm{IC}_{50}=39 \mu \mathrm{M}, \quad \Delta \Delta \mathrm{G}_{\text {bind }}=-3.10 \mathrm{kcal} / \mathrm{mol}$, Table 1). It can therefore be predicted that Nutlin-3A has an effect on WD/DD LPS with high levels of full-length MDM2 proteins, but not in the presence of high levels of MDM2-B or MDM2-C proteins and that the exact estimation of MDM2 full-length and MDM2-B and MDM2-C protein isoforms can allow an accurate prediction of the drug efficacy.

\section{MDMX}

\section{FISH}

FISH analysis revealed that all of the examined specimens showed an MDMX disomic pattern in both the WD and DD components (data not shown).

\section{Table 1 Calculated free energy of binding $\Delta \mathbf{G}_{\mathrm{bind}}, \mathrm{IC}_{50}$, and $\Delta \Delta \mathbf{G}_{\text {bind }}$ values for Nutlin-3A in complex with MDM2 and MDMX, and their splicing variants MDM2-B, MDM2-C, and MDMX-S}

\begin{tabular}{lccc}
\hline Protein & $\Delta G_{\text {bind }}(\mathrm{kcal} / \mathrm{mol})$ & $\mathrm{IC}_{50}(\mathrm{mM})$ & $\Delta \Delta \mathrm{G}_{\text {bind }}(\mathrm{kcal} / \mathrm{mol})^{\mathrm{ab}}$ \\
\hline MDM2 & $-9.11 \pm 0.23$ & 0.21 & - \\
MDM2-B & $-6.01 \pm 0.27$ & 39 & -3.10 \\
MDM2-C & $-7.03 \pm 0.19$ & 7.1 & -2.08 \\
MDMX & $-7.10 \pm 0.24$ & 6.1 & - \\
MDMX-S & $-8.06 \pm 0.25$ & 1.2 & +0.96
\end{tabular}

${ }^{a} \Delta \Delta G_{\text {bind }}$ is defined as $\Delta \Delta G_{\text {bind }}=\Delta G_{\text {bind }}$ (native protein) $-\Delta G_{\text {bind }}$ (splicing variant). A negative $\Delta \Delta G_{\text {bind }}$ value indicates greater Nutlin-3A affinity for the native protein than for the splicing variant, whereas a positive $\Delta \Delta G_{\text {bind }}$ value indicates greater Nutlin-3A affinity for the splicing variant.

${ }^{\mathrm{b}} \mathrm{A}$ difference of $1.4 \mathrm{kcal} / \mathrm{mol}$ from $\Delta \mathrm{G}_{\text {bind }}$ corresponds to a one order of magnitude increase in the affinity of Nutlin-3A for a given protein. 
a

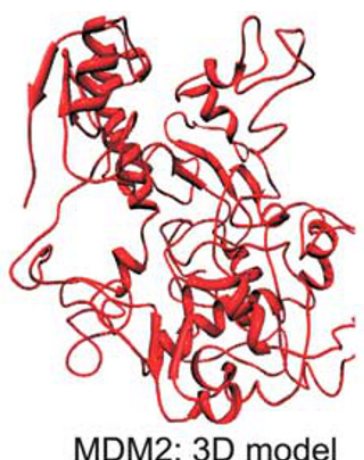

C

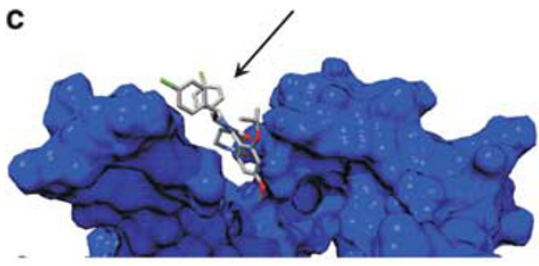

MDM2-B: Nutlin-3A IC ${ }_{50}=39 \mu \mathrm{M}$ b
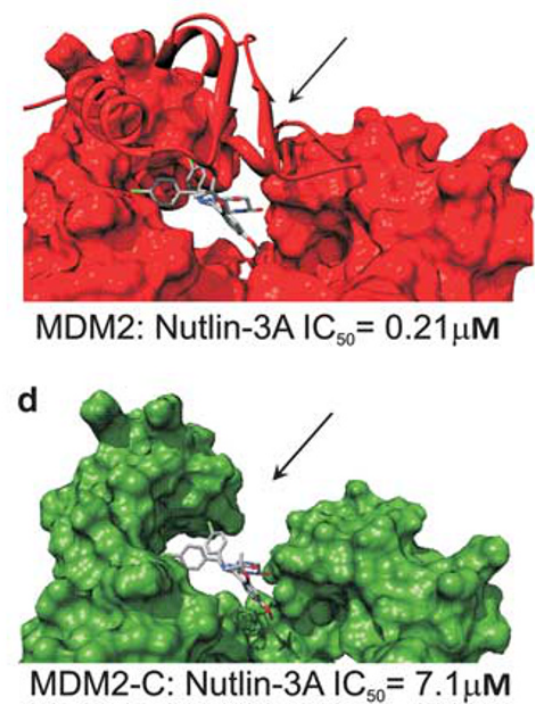

Figure 2 (a) Three-dimensional model of full-length MDM2. Predicted mode of Nutlin-3A binding to full-length MDM2 (b), MDM2-B (c), and MDM2-C (d) splicing variants. In panels (b-d), the protein is represented by its colored van der Waals surface and Nutlin-3A as atom-colored sticks (C, gray; $\mathrm{O}$, red; $\mathrm{N}$, blue; $\mathrm{Cl}$, green). Hydrogen, water molecules, ions, and counter-ions have been omitted for the sake of clarity. In panel (b), the part of the $\mathrm{N}$-terminal domain of MDM2 that is missing in the larger MDM2 splicing variant (MDM2-C) is highlighted.

To evaluate further the potential use of Nutlin-3A in treating WD/DD LPS, the analyses were extended to MDMX and its splicing variant MDMX-S.

\section{MDMX Transcript Detection and Quantification}

Previously described specific primers ${ }^{22}$ were used to coamplify full-length $m d m x$ and $m d m x-S$ transcripts, and the PCR products were cloned and sequenced (Supplementary Figure 3). As shown in Figures $3 \mathrm{a}$ and $\mathrm{b}$, both full-length $m d m x$ and $m d m x$-s transcripts were present in all of the WD/ DD samples. The relative amounts of the full-length $m d m x$ and $m d m x$-s transcripts were evaluated by means of densitometry on agarose gel: the ratio in the tumor samples ranged from 0.1 (ie $10 \%$ of $m d m x-s$ transcript and $90 \%$ of the fulllength $m d m x$ transcript) to the unexpected level of 5.6 (ie $85 \%$ of the $m d m x-s$ transcript and $15 \%$ of the $m d m x$ transcript). These findings indicate that (as previously reported for various cell lines), ${ }^{19}$ the amount of $m d m x-s$ transcript varied widely from one sample to another and, in some cases (three WD and three DD LPS), exceeded that of $m d m x$. Taking into account the limited number of samples examined, we cannot rule out that elevated $m d m x-s / m d m x$ transcript ratios can be present in both WD and DD variants.

\section{MDMX Protein Expression}

Stronger MDMX nuclear than cytoplasmic immunostaining has been found in various tumor histotypes ${ }^{32-34}$ and, despite its lack of a nuclear signal localization domain (Supplementary Figure 3), ${ }^{35}$ it has also been reported that MDMX-S localizes in the nucleus and the cytoplasm. ${ }^{16}$ Using an antibody that recognizes only full-length MDMX, stronger nuclear than cytoplasmic labeling was demonstrated by means of IHC in matched FFPE samples of both the WD and the DD components (Figure 3c).

In order to extend the IHC results, MDMX and MDMX-S were analyzed by means of WB. The actual size of MDMX-S is still controversial and so, to shed further light on the question, the transcript corresponding to human MDMX-S was amplified by PCR and cloned in PCDNA3, and the size of MDMX-S was estimated on the corresponding IVT product and the protein produced in $293 \mathrm{~T}$ cells by transient transfection. In both instances, immunoblotting with an MDMXS-specific antibody ${ }^{19}$ (MX-82, Sigma) revealed a band of $\sim 18 \mathrm{kDa}$ (Supplementary Figure 4). In line with this, a band of $18 \mathrm{kDa}$ (Figure 3d) corresponding to the molecular weight of MDMX-S was identified in most of the WD/DD samples, together with an $80-\mathrm{kDa}$ band corresponding to full-length MDMX protein.

Although it has been suggested that $m d m x-s$ RNA may act as a means of reducing full-length MDMX levels rather than being translated into protein, ${ }^{20}$ we found that the $m d m x$-s transcript in our WD/DD LPS samples was translated into protein, albeit to varying extents.

\section{Sensitivity of TP53/MDMX and TP53/MDMX-S Complexes to} Nutlin-3A

The ability of Nutlin-3A to affect the binding of MDMX and MDMX-S to TP53 was evaluated by means of GST pull-down 
a

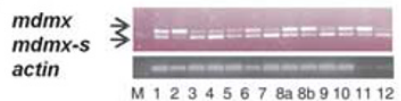

b

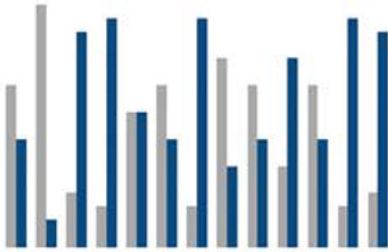

Cases $\quad \begin{array}{llllllllllllll}1 & 2 & 3 & 4 & 5 & 6 & 7 & 8 a & 8 b & 9 & 10 & 11 & 12\end{array}$

MDMX.S $40 \quad 10808550408530 \quad 4070408580$

MDMX $\quad 609020 \quad 155060 \quad 1570 \quad 6030 \quad 6015 \quad 20$

ratio $\quad 0.70 .14 \quad 5.61 \quad 0.75 .60 .40 .72 .50 .75 .64$

c

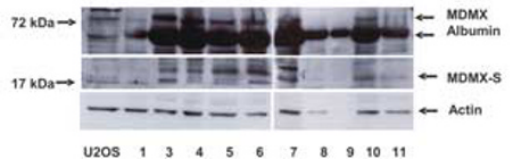

d

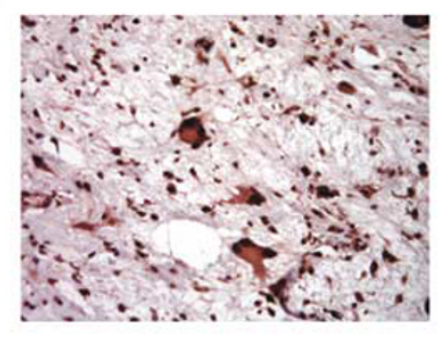

e

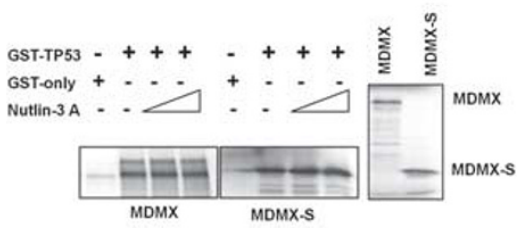

Figure 3 (a) Representative $m d m x$ WD/DD LPS RT-PCR results. The $m d m x$ and $m d m x-s$ transcripts are indicated by the arrows. (b) The densitometry analysis of the (gray columns) and $m d m x-s$ (blue columns) transcripts was made as described for $m d m 2-b / m d m 2$. The $m d m x-s / m d m x$ ratio was obtained in the same way as the $m d m 2 / m d m 2-b$ ratio. (c) IHC analysis of a representative WD/DD sample. Both nuclear and cytoplasmatic staining were present. (d) WB analysis of six representative WD/DD samples. The arrows indicate the bands corresponding to MDMX (80 kDa) and MDMX-S (18 kDa) (also see Supplementary Figure 4). (e) Complexes of TP53 and MDMX splice variants are refractory to Nutlin-3A inhibition. GST pull-down assay of GST-TP53 with IVT-MDMX or MDMX-S. GST was used alone as the negative control. The experiments were carried out in the absence (-) or presence of increasing amounts of Nutlin-3A. The panel on the right shows $10 \%$ input.

assays. As shown in Figure 3e, both splice variants efficiently interact with TP53, and these interactions are resistant to disruption by Nutlin-3A.

\section{MDMX and MDMX-S Molecular Modeling}

MM/PBSA MD simulations were performed using MDMX and MDMX-S in complex with Nutlin-3A. Importantly, our in silico results reproduced experimental evidence ${ }^{17}$ that MDMX binding requires a much higher concentration of Nutlin-3A $\left(\mathrm{IC}_{50}=6.1 \mu \mathrm{M}, \Delta \mathrm{G}_{\text {bind }}=-7.10 \mathrm{kcal} / \mathrm{mol}\right.$, Table 1) than MDM2 binding $\left(\mathrm{IC}_{50}=0.21 \mu \mathrm{M} \Delta \mathrm{G}_{\mathrm{bind}}=-9.11 \mathrm{kcal} /\right.$ mol, Table 1). The difference in Nutlin-3A affinity for the two oncogenes is rooted in the drug binding site that, despite the sequence homology of the two proteins, is narrower in MDMX than in MDM2, and therefore creates greater steric hindrance to drug binding (compare panels $\mathrm{a}$ and $\mathrm{b}$ in Figure 4). The simulations also revealed that Nutlin-3A binds to MDM2 in its 'closed' state: ie when the N-terminal lid of MDM2 (yellow ribbon in Figure 4a) is folded onto the TP53 binding cleft. In the case of MDMX, the amino-acid sequence of the lid motif is different, and so the conformation of the closed lid state (yellow ribbon in Figure $4 \mathrm{~b}$ ) has to be perturbed to allow Nutlin-3A binding, thus leading to a free binding energy penalty.

In the case of the MDMX-S splicing variant, our simulations predicted that its affinity would be slightly higher than that of full-length $\operatorname{MDMX}\left(\Delta \Delta \mathrm{G}_{\text {bind }}=+0.96 \mathrm{kcal} / \mathrm{mol}\right.$,
Table 1). The predicted fold of MDMX-S (see Figure 4c) indicates that, although the $\mathrm{N}$-terminal lid and drug binding site are fully preserved in the truncated protein, their overall conformation undergoes small readjustments that ultimately lead to a slightly less tight, and therefore less hindered, binding site for TP53 (and consequently Nutlin-3A; yellow ribbon in Figure 4d). This different binding site conformation also implies that some residues are more efficient in lining the binding pocket with their side-chains in the case of MDMX-S, thus increasing the number of favorable stabilizing (eg hydrophobic) contacts with the drug (detailed in Figure 4e). Accordingly, Nutlin-3A is five times more potent in inhibiting MDMX-S $\left(\mathrm{IC}_{50}=1.2 \mu \mathrm{M}\right)$ than MDMX $\left(\mathrm{IC}_{50}=6.1 \mu \mathrm{M}\right)$, although its potency is still two orders of magnitude lower than that observed in the case of MDM2.

Confirmation of the differential affinity of Nutlin-3A for MDM2, MDMX, and their splicing variants was obtained using SMD simulations of all of the Nutlin-3A/protein complexes. Mimicking the drug unbinding process, we used SMD-derived force profiles to distinguish tight from loose Nutlin-3A binding to all of the proteins. As clearly shown in Figure 5, stronger forces $(\sim 800 \mathrm{pN})$ and longer times ( $\sim 400 \mathrm{ps}$ ) are required to detach Nutlin-3A from its MDM2 binding site, whereas considerably lower intensities ( $\sim 250$ $400 \mathrm{pN}$ ) and shorter times ( $\sim 200 \mathrm{ps})$ are needed to unbind the drug from MDMX and all of the splicing variants under the same conditions. 

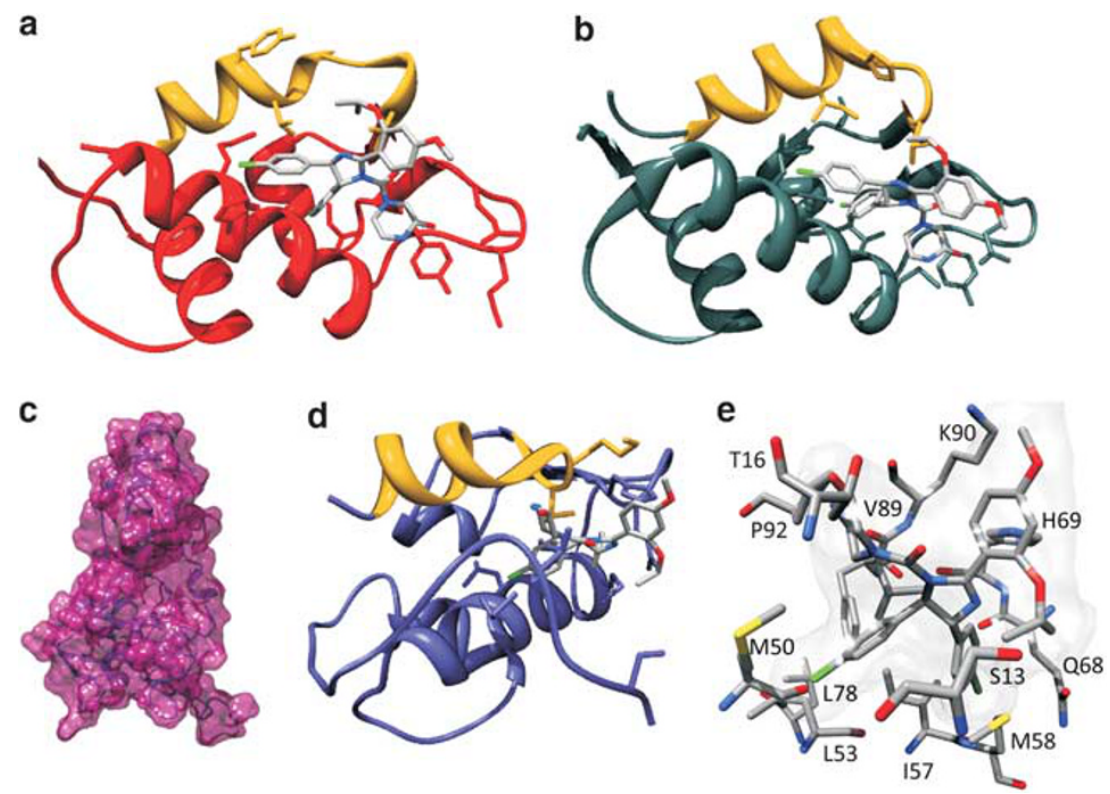

Figure 4 Zoomed view of Nutlin-3A in complex with full-length (FL) MDM (a) and MDMX (b). (c) Three-dimensional model of MDMX-S. The protein's secondary structure is shown as a lavender ribbon, and its van der Waals surface is shown in dark violet. (d) Zoomed view of Nutlin-3A in complex with MDMX-S. (e) Details of the interactions between MDMX-S residues and Nutlin-3A. In panels (a), (b), and (d), the protein structures are shown as red, gray, and slate blue ribbons; the $\mathrm{N}$-terminal lid motif is in yellow. The protein residues lining the binding sites are shown as correspondingly colored sticks; Nutlin-3A is shown as atom-colored sticks ( $\mathrm{C}$, gray; $\mathrm{O}$, red; $\mathrm{N}$, blue; $\mathrm{Cl}$, green). In panel (E), some protein residues and the drug are highlighted as atom-colored sticks ( $\mathrm{C}$, gray; $\mathrm{O}$, red; $\mathrm{N}$, blue; $\mathrm{Cl}$, green; $\mathrm{S}$, yellow). Some of the main protein residues interacting with Nutlin-3A are labeled. Hydrogen, water molecules, ions, and counter-ions have been omitted for the sake of clarity.

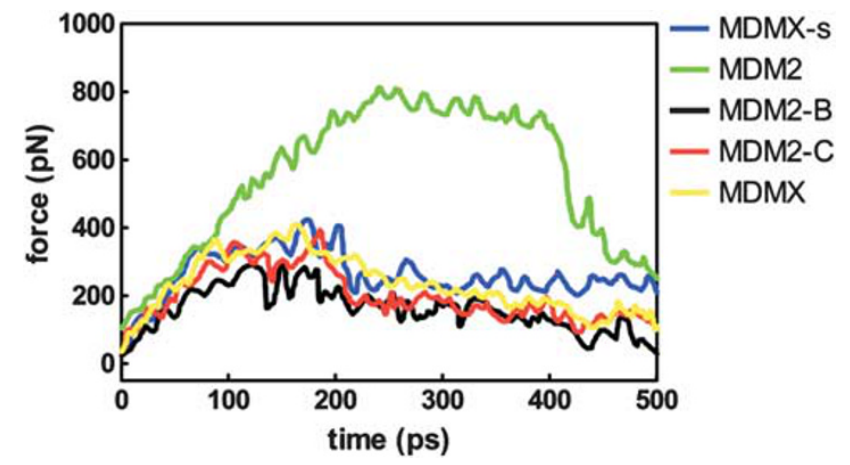

Figure 5 Comparison of the unbinding force profiles of Nutlin-3A from full-length MDM2 (green), MDM2-B (black), MDM2-C (red), MDMX (yellow), and MDMX-S (blue). The plots of each protein show the mean values obtained by averaging the force profiles of five different SMD runs.

\section{DISCUSSION}

We used RT-PCR to interrogate a series of cryopreserved samples obtained from surgical specimens, and found that full-length and alternative transcripts and splicing isoforms of both MDM2 and MDMX pertain to the profile of WD/DD LPS. Subsequently, using biochemical analysis and in vitro assays complemented by molecular modeling, we investigated whether the transcripts that were translated into proteins were differently targeted by Nutlin-3A.
As expected, FISH showed that the MDM2 gene was amplified in the WD and DD components of all of our cases $^{36}$ and, as previously reported, ${ }^{12}$ sequencing identified SNP309 (GG allele) in half of the WD/DD samples. Broadening the analysis to the transcripts, we found presence of full-length $m d m 2$ in all of the cases and the alternative $m d m 2-b$ splicing form in the overwhelming majority of samples (making a similar contribution to both the WD and DD components) (Figure 1a). On the contrary, the $m d m 2-c$ transcript was identified in only one sample of each component, and there were no transcripts corresponding to $m d m 2-a$. Subsequent WB analysis (Figure 1c) showed that, of the three isoforms lacking the TP53 binding site ( $m d m 2-a, m d m 2-b$, and $m d m 2-c$ ), only $m d m 2-b$ transcripts were translated into protein (at least at our level of detection).

Analyses assessing the ability of Nutlin-3A to inhibit the formation of TP53/MDM2 complexes, and the energy needed to form MDM2/Nutlin-3A complex, gave similar results. GST pull-down assays showed that MDM2-B and MDM2-C, which cannot bind TP53, were unaffected by Nutlin-3A, and molecular modeling revealed that the affinity of MDM2-B for Nutlin-3A was two orders of magnitude less than that for the native protein $(0.21 v s 39 \mu \mathrm{M}$; Figure 2 and Table 1). It was also predicted that MDM2-C has a partially negative effect on Nutlin-3A and that MDM2-A made an irrelevant contribution (Figure 2 and Table 1). 
Taken together, these findings predict that the response of WD/DD LPS to Nutlin-3A depends on the amount of the coexpressed MDM2-B isoform. The lower affinity of MDM2-B, which was further confirmed by MD simulations, is keeping with preclinical in vitro and in vivo data ${ }^{37}$ and supports the idea that MDM2-B may contribute to the formation of cancer by means of a TP53-independent mechanism. ${ }^{38}$ In particular, transduction of MDM2-B into a variety of cell types reveals that MDM2-B promotes TP53-independent cell growth, inhibits apoptosis, and upregulates the RelA subunit of $\mathrm{NF}_{\mathrm{k}} \mathrm{B} .{ }^{38}$ and that is expected to correlate with a more aggressive behavior. In line with recent sarcoma genetic data, ${ }^{22}$ all of the analyzed WD/DD LPS were disomic for MDMX.

Transcript analysis showed that full-length $m d m x$ was expressed together with the $m d m x$-s splicing variant in all of the cases, and similarly in the WD and DD components (Figure 3), and it is worth noting that the level of $m d m x-s$ expression was in some cases (three WD and three DD LPS) higher than that of $m d m x$. Moreover, quantitative analysis (Figure 3) showed that the $m d m x-s / m d m x$ ratio ranged from 0.25 to 5.6, and that the corresponding estimated $m d m x-s$ rate ranged from 20 to $85 \%$. IHC and WB confirmed that the transcripts were translated into proteins (Figure 3).

The GST pull-down assays demonstrated that Nutlin3A was unable to affect the binding of TP53 to MDMX or MDMX-S significantly (Figure 3). Molecular modeling supported these results by predicting that the affinity of Nutlin-3A for MDMX and MDMX-S was two orders of magnitude lower than that for MDM2, which suggests that Nutlin-3A may not be very efficacious in WD/DD LPS patients.

As drug efficacy and its expected impact on clinical results are greatly strengthened by measuring the force and time that is necessary to detach Nutlin-3A from its binding sites, we used SMD to mimic the drug unbinding process. As clearly shown in Figure 5, these parameters (which are directly proportional to drug affinity) decrease from MDM2 to MDMX-S to MDMX and, lastly, to MDM2-B. To our knowledge, all the WD/DD established cell lines are not commercially available, however, it could be interesting evaluating the Nutlin-3A efficacy after ectopic induction of MDM2-B, MDM2-C and MDMX-S expressions.

The overall results of this study provide some interesting new insights into the molecular profile of WD/DD LPS that not only help to explain their variable sensitivity to Nutlin$3 \mathrm{~A}$ in vitro and in the clinical setting, but also support the development of dual MDM2/MDMX compounds ${ }^{38}$ that are expected to improve clinical outcomes in non-resectable WD/DD patients.

Supplementary Information accompanies the paper on the Laboratory Investigation website (http://www.laboratoryinvestigation.org)

Supported by AIRC grants to SP and RM.

\section{DISCLOSURE/CONFLICT OF INTEREST}

The authors declare no conflict of interest.

1. Mariño-Enríquez $A$, Fletcher $C D$, Dal Cin $P$, et al. Dedifferentiated liposarcoma with "homologous" lipoblastic (pleomorphic liposarcomalike) differentiation: clinicopathologic and molecular analysis of a series suggesting revised diagnostic criteria. Am J Surg Pathol 2010;34: 1122-1131.

2. Boland JM, Weiss SW, Oliveira AM, et al. Liposarcomas with mixed well-differentiated and pleomorphic features: a clinicopathologic study of 12 cases. Am J Surg Pathol 2010;34:837-843.

3. Singer $\mathrm{S}$, Antonescu CR, Riedel $\mathrm{E}$, et al. Istologic subtype and margin of resection predict pattern of recurrence and survival for retroperitoneal liposarcoma. Ann Surg 2003;238:358-370.

4. Fletcher CDM, Bridge JA, Hegendoorn PCW, et al. World Health Organization Classification of Tumors of Soft Tissue and Bone. 4th edn, (Lyon, 2013) pp37-38.

5. Butò $S$, Pierotti MA, Tamborini $E$, et al. Biochemical uncovering of $\mathrm{mdm} 2 / \mathrm{p} 53$ complexes in liposarcomas parallels their immunohistochemical detection. Diagn Mol Pathol 1999;8:125-130.

6. Pilotti S, Della Torre G, Lavarino C, et al. Distinct mdm2/p53 expression patterns in liposarcoma subgroups: implications for different pathogenetic mechanisms. J Pathol 1997;181:14-24.

7. Pilotti S, Della Torre G, Lavarino C, et al. Molecular abnormalities in liposarcoma: role of MDM2 and CDK4-containing amplicons at 12q13-22. J Pathol 1998;185:188-190.

8. Vassilev LT, Vu BT, Graves B, et al. In vivo activation of the p53 pathway by small-molecule antagonists of MDM2. Science 2004;303: 844-848.

9. Shangary S, Wang S. Small-molecule inhibitors of the MDM2-p53 Protein-Protein interaction to reactivate p53 function: a novel approach for cancer therapy. An Rev Pharmacol Toxicol 2009;49: 223-241.

10. Tamborini E, Della Torre G, Lavarino C, et al. Analysis of the molecular species generated by MDM2 gene amplification in liposarcomas. Int J Cancer 2001;92:790-796.

11. Sigalas I, Calvert AH, Anderson JJ, et al. Alternatively spliced mdm2 transcripts with loss of p53 binding domain sequences: transforming ability and frequent detection in human cancer. Nat Med 1996;2: 912-917.

12. Ito $M$, Barys $L, O^{\prime}$ Reilly $T$, et al. Comprehensive mapping of $p 53$ pathway alterations reveals an apparent role for both SNP309 and MDM2 amplification in sarcomagenesis. Clin Cancer Res 2011;17: 416-426.

13. Seyfried I, Hofbauer S, Stoecher M, et al. SNP309 as a predictor for sensitivity of CLL cells to the MDM2 inhibitor nutlin-3a. Blood 2008; 112:2168.

14. Malek SM. MDM2-SNP 309 allele does not affect sensitivity to MDM2 inhibitors in CLL. Blood 2008;112:2169.

15. Linares LK, Hengstermann A, Ciechanover A, et al. HdmX stimulates Hdm2-mediated ubiquitination and degradation of p53. Proc Natl Acad Sci USA 2003;100:12009-12014.

16. Patton JT, Mayo LD, Singhi $A D$, et al. Levels of $H d m X$ expression dictate the sensitivity of normal and transformed cells to Nutlin-3. Cancer Res 2006:66:3169-3176.

17. Hu B, Gilkes DM, Farooqi B, et al. MDMX overexpression prevents p53 activation by the MDM2 inhibitor Nutlin. J Biol Chem 2006;281: 3303-3305.

18. Rallapalli R, Strachan $G$, Tuan RS, et al. Identification of a domain within MDMX-S that is responsible for its high affinity interaction with p53 and high-level expression in mammalian cells. J Cell Biochem 2003;89:563-575.

19. Lenos $K$, Jochemsen AG. Functions of MDMX in the modulation of the p53-response. J Biomed Biotechnol 2011;2011:876173.

20. Lenos K, Grawenda AM, Lodder K, et al. Alternate splicing of the p53 inhibitor HDMX offers a superior prognostic biomarker than p53 mutation in human cancer. Cancer Res 2012;72: 4074-4084.

21. Müller $C R$, Paulsen EB, Noordhuis $P$, et al. Potential for treatment of liposarcomas with the MDM2 antagonist Nutlin-3A. Int J Cancer 2007;121:199-205. 
22. Bartel F, Schulz J, Böhnke A, et al. Significance of HDMX-S (or MDM4) mRNA splice variant overexpression and HDMX gene amplification on primary soft tissue sarcoma prognosis. Int J Cancer 2005;117:469-475.

23. Tamborini E, Casieri P, Miselli F, et al. Analysis of potential receptor tyrosine kinase targets in intimal and mural sarcomas. J Pathol 2007;212:227-235.

24. Piccinin S, Tonin E, Sessa S, et al. A "twist box" code of p53 inactivation: twist box: p53 interaction promotes p53 degradation. Cancer Cell 2012;22:404-415.

25. Poyurovsky MV, Katz C, Laptenko $\mathrm{O}$, et al. The C terminus of p53 binds the N-terminal domain of MDM2. Nat Struct Mol Biol 2010; 17:982-989.

26. Pierotti MA, Negri T, Tamborini $E$, et al. Targeted therapies: the rare cancer paradigm. Mol Oncol 2010;4:19-37.

27. Ferrone M, Perrone F, Tamborini E, et al. Functional analysis and molecular modeling show a preserved wild-type activity of p53 (C238Y). Mol Cancer Ther 2006;5:467-473.

28. Srinivasan J, Cheatham III TE, Cieplak $\mathrm{P}$, et al. Continuum solvent studies of the stability of DNA, RNA, and phosphoroamidate-DNA helices. J Am Chem Soc 1998;120:9401-9409.

29. Isralewitz B, Gao M, Schulten K. Steered molecular dynamics and mechanical functions of proteins. Curr Opin Struct Biol 2001;11:224-230.

30. Ohnstad HO, Paulsen EB, Noordhuis P, et al. MDM2 antagonist Nutlin3a potentiates antitumour activity of cytotoxic drugs in sarcoma cell lines. BMC Cancer 2011;11:1-11.
31. Shaomeng W, Yujun Z, Denzil B, et al. Targeting the MDM2-p53 protein-protein interaction for new cancer therapeutics. Top Med Chem 2012;8:57-80.

32. Han X, Garcia-Manero G, McDonnell TJ, et al. HDM4 (HDMX) is widely expressed in adult pre-B acute lymphoblastic leukemia and is a potential therapeutic target. Mod Pathol 2007;20: 54-62.

33. Liang M, Han X, Vadhan-Raj S, et al. HDM4 is overexpressed in mantle cell lymphoma and its inhibition induces p21 expression and apoptosis. Mod Pathol 2010;23:381-391.

34. Valentin-Vega YA, Barboza JA, Chau GP, et al. High levels of the p53 inhibitor MDM4 in head and neck squamous carcinomas. Hum Pathol 2007;38:1553-1562.

35. Mancini F, Conza GD, Moretti F. MDM4 (MDMX) and its transcript variants. Curr Genomics 2009;10:42-50.

36. Horvai $A E$, DeVries $S$, Roy $R$, et al. Similarity in genetic alterations between paired well-differentiated and dedifferentiated components of dedifferentiated liposarcoma. Mod Pathol 2009;22:1477-1488.

37. Steinman HA, Burstein E, Lengner C, et al. An alternative splice form of Mdm2 induces p53-independent cell growth and tumorigenesis. J Biol Chem 2004;279:4877-4886.

38. Graves B, Thompson $\mathrm{T}$, Xia M, et al. Activation of the p53 pathway by small-molecule-induced MDM2 and MDMX dimerization. Proc Nat Acad Sci USA 2012;109:11788-11793. 